\title{
Internationale Aktivitäten in Zusammenhang mit Sedimentforschung und -management auf globaler, europäischer, regionaler und Einzugsgebietsebene
}

\author{
Helmut Habersack · Doris Gangl · Angelika Riegler · Mario Klösch · Philipp Gmeiner · Marlene Haimann
}

Online publiziert: 6. Februar 2019

(c) Der/die Autor(en) 2019

Zusammenfassung Sedimentüberschuss und -defizit als Folge der Unterbrechung des Sedimentkontinuums, der Erhöhung der Transportkapazität durch Regulierungsmaßnahmen mit Reduktion der Sohlbreite, der Erhöhung des Sohlgefälles und Unterbindung der Seitenerosion und Umlagerungen sowie der Abtrennung von Überflutungsflächen nehmen in nahezu allen Flussgebieten zu. Landnutzungsänderungen und Klimawandel (z.B. Abschmelzen der Gletscher) können diese Tendenz verschärfen. Die Folgen sind Stauraumverlandungen, die trotz enormer Investitionen in die Errichtung neuer Anlagen zu einer Nettoabnahme des weltweiten Stauvolumens führen und in den freien Fließstrecken Sohleintiefungen hervorrufen. Dies reicht bis hin zu Küstenerosionen in signifikantem Ausmaß, wenn nur mehr deutlich geringere Sedimentmengen das Meer erreichen. Die Folgen der Änderungen der Sedimentbilanz können zu einer negativen Beeinflussung der Wasserkraft, Erhöhung des Hochwasserrisikos, Verschlechterung der Schifffahrtsbedingungen oder negativen Auswirkungen auf den ökologischen Zustand der Flüsse führen. Daraus ergibt sich der Bedarf, verstärkt Sedimentforschung und -management zu betreiben. Dieser Artikel behandelt dazu internationale Aktivitäten auf globaler, europäischer, regionaler und Einzugsgebietsebene. Global werden beispielsweise die sedi-

Univ.-Prof. DI Dr. H. Habersack ( $\bowtie)$. Mag. Dr. D. Gangl · Mag. A. Riegler · DI M. Klösch · DI P. Gmeiner DI M. Haimann

Institut für Wasserbau, Hydraulik und Fließgewässerforschung, Christian Dopplerlabor für Sedimentforschung und -management, Department Wasser - Atmosphäre - Umwelt, Universität für Bodenkultur Wien, Muthgasse 107, 1190 Wien, Österreich helmut.habersack@boku.ac.at mentbezogenen Aktivitäten der UNESCO IHP World's Large Rivers Initiative (WLRI), der International Sediment Initiative (ISI), der International Association for Hydro-Environment Engineering and Research (IAHR) und der World Association for Sedimentation and Erosion Research (WASER) vorgestellt. Auf europäischer Ebene geht es um das European Sediment Network (SedNet), auf regionaler Ebene um die INTERREG-Alpenraumprojekte SedAlp und HyMoCARES. Das EUSDR Flagship-Projekt Danube River Research and Management (DREAM), das INTERREG DTP-Projekt DanubeSediment und drei bilaterale INTERREG-Projekte betreffen das Donaueinzugsgebiet. Schließlich wird noch auf einzelne Sedimentaktivitäten an Rhein und Elbe eingegangen, an deren Einzugsgebiet Österreich Anteil hat.

Schlüsselwörter Sedimente

Geschiebe · Schwebstoffe .

Feststoffhaushalt · Flussmorphologie .

Sohleintiefung · Stauraumverlandung

International activities related to sediment research and management at global, European, regional and catchment scale

Abstract Sediment surplus and deficit following the interruption of sediment continuity, the increase of the transport capacity due to river regulation leading to a reduction of the river bed width, an increase of bed slope and prohibition of side erosion and bed form development as well as cutting off floodplains are growing in almost all river basins. Land use change and climate change (e.g. melting of glaciers) can intensify this tendency. Consequences are the sedimentation of reservoirs, which lead-despite huge investments to construct new dams-to a net reduction of the global storage volume und river bed erosion in the free flowing river sections. This leads ultimately to significant coastal erosion when considerably less sediments are reaching the sea. These changes of the sediment balance negatively influence hydropower, increase floodrisk, worsen navigation conditions or the ecological status. This leads to an increasing demand for sediment research and management. This article presents international activities at global, European, regional and catchment scale. Globally the sediment work of the UNESCO IHP World's Large Rivers Initiative (WLRI), the International Sediment Initiative (ISI), the International Association for Hydro-Environment Engineering and Research (IAHR) and the World Association for Sedimentation and Erosion Research (WASER) are described. The European Sediment Network (SedNet) is representing an activity in Europe, at regional scale the INTERREG Alpine Space projects SedAlp and HyMoCARES are introduced. The EUSDR Flagship Project Danube River Research and Management (DREAM), the INTERREG DTP Project DanubeSediment and three bilateral INTERREG projects concern the Danube basin. Finally, sediment activities at the Rhine and Elbe, where Austria has a share of the catchment area, are presented.

Keywords Sediments - Bedload . Suspended load · Solid budget · River morphology · River bed erosion . Reservoir sedimentation

\section{Einleitung}

Sedimentforschung und -management kommt nicht nur national, sondern auch international immer stärkere Bedeutung zu. Dies wird durch eine große Anzahl an Aktivitäten in diesem Themenfeld dokumentiert. Vor allem die anwendungsorientierten Themenstellungen in Zusammenhang mit der Nutzung und dem Schutz von Fließgewäs- 
sern fokussieren stärker darauf, da die Probleme in den letzten Jahrzehnten tendenziell zugenommen haben. Andererseits hat die Grundlagenforschung im Bereich des Monitorings und der Modellierung signifikante Fortschritte gemacht (Liedermann et al. 2019, in diesem Heft; Tritthart et al. 2019, in diesem Heft), sodass Probleme aufgezeigt werden konnten, aber zunehmend auch Maßnahmen zur Lösung dieser Probleme erarbeitet wurden (Hauer et al. 2019, in diesem Heft; Schobesberger et al. 2019, in diesem Heft).

Die praktischen Probleme resultieren aus der zunehmenden Schere zwischen Überschuss und Defizit an Sedimenten (Habersack et al. 2017). Während beispielsweise die Anhebung der Alpen weitergeht (Nocquet et al. 2016) und auch die Verwitterung und somit Bereitstellung von Material voranschreitet, kommen durch den Klimawandel voraussichtlich zusätzliche Mengen in das System. Das ist durch das Abschmelzen der Gletscher und das Zurückgehen des Permafrostes bedingt. Darüber hinaus erfolgt eine intensive Landnutzungsänderung, die global gesehen in vielen Regionen und Flusseinzugsgebieten z.B. eine Abholzung der Regenwälder, Intensivierung der Landwirtschaft und Erhöhung der Bodenerosion und damit Eintrag in Flusssysteme samt des damit in Verbindung stehenden Nährstoff- und Schadstoffeintrages zur Folge hat (Gupta 2008; Best 2019).

Auf globaler Ebene werden pro Jahr ca. 12,6 Gt Sedimente ins Meer eingetragen, $14 \mathrm{Gt}$ waren es vor anthropogenen Einflüssen, $24 \mathrm{Gt}$ Reduktion resultiert aus Stauraumverlandung. Das ergibt 36,6 Gt ohne Stauraumverlandung, was ca. $160 \%$ Steigerung gegenüber dem unbeeinflussten System und ca. $66 \%$ Reduktion wegen der Verlandung ausmachen würde (Walling 2008, 2015). Ein Management dieser Mengen ohne „Mithilfe“ der Flüsse wäre weder technisch noch ökonomisch oder ökologisch möglich. Darüber hinaus steht in vielen Ländern die Frage im Raum, ob Sedimente - wenn sie einmal dem Fluss entnommen wurden - als Abfall $\mathrm{zu}$ sehen sind und damit eine entsprechende Behandlung bis hin zu einer Deponierung erfahren müssen. Dazu wird derzeit im Bereich der Wildbachund Lawinenverbauung in Österreich ein ÖWAV-Regelblatt ausgearbeitet, wo u.a., wenn möglich, die Priorität auf dem Halten der Sedimente im System liegt (Habersack et al. 2018).

Im Bereich der Wasserkraftnutzung, der Wildbachverbauung aber teilweise auch z.B. bei Rückhaltebecken im Hochwasserrisikomanagement dominiert der Überschuss an Sedimenten (Bock et al. 2019, in diesem Heft). Dieser führt zur Reduktion von Stauraumvolumina und damit Reduktion der Stromproduktion bis hin zu Standsicherheitsproblemen der Wasserbauwerke.

Demgegenüber gibt es in den meisten freien Fließstrecken ein Sedimentdefizit, das mittlerweile in vielen Mündungsbereichen von Flüssen zu Küstenerosionen (Habersack et al. 2017) oder Absenkungen von Flussdeltas führt (Kondolf et al. 2018). Neben dem Landverlust steigt dort auch die Anfälligkeit gegenüber der Erhöhung des Meeresspiegels infolge des Klimawandels.

Dieser Artikel zeigt einige internationale Aktivitäten betreffend Sedimentforschung und -management - großteils mit Beteiligung der AutorInnen auf und soll darauf aufmerksam machen, dass dieses Thema beim Fließgewässermanagement künftig stärkere Beachtung finden sollte.

\section{Globale Aktivitäten in Zusammenhang mit Sedimentforschung und -management}

2.1 UNESCO IHP World's Large Rivers Initiative und International Sediment Initiative

Das International Hydrological Programme (IHP) der UNESCO ist im UNSystem das einzige zwischenstaatliche Programm, welches sich mit dem Thema Wasser wissenschaftlich auseinandersetzt und auch die Bildung und den Wissenstransfer im Fokus hat (https:// en.unesco.org/themes/water-security/ hydrology). IHP wurde 1975 gegründet und derzeit erfolgt die Implementierung der achten Phase „Water Security“. Als Vizepräsident des Zwischenstaatlichen Rates, zuständig für Westeuropa und Nordamerika, erfolgten durch den Erstautor erste Weichenstellungen für das neunte Programm und die Weiterentwicklung des IHP. Über den an der BOKU angesiedelten UNESCO Chair „Integrated River Research and Management“ wird u. a. die „World's Large Rivers Initiative“ koordiniert (http:// worldslargerivers.boku.ac.at/wlr/).
Hier geht es um eine integrative Betrachtung von Flüssen, wobei aufgrund der globalen Skala ein Fokus auf große Flüsse gelegt wird. Dabei bedeutet groß ein Mittelwasserdurchfluss größer $2000 \mathrm{~m}^{3} / \mathrm{s}$ an der Mündung, ein Einzugsgebiet größer $500.000 \mathrm{~km}^{2}$ oder eine Flusslänge größer $1000 \mathrm{~km}$ (gemessen am Hauptarm). Um einen inklusiven Prozess zu gewährleisten, können andere quantitative und qualitative Kriterien herangezogen werden, z.B. relative Maße, bezogen auf die Größe des Landes (z.B. die natürliche, kulturelle und ökonomische Bedeutung des jeweiligen Flusses).

Da es zurzeit keinen einheitlichen Statusbericht zum Zustand der großen Flüsse der Welt gibt, wurde 2012 die World's Large Rivers Initiative (WLRI) ins Leben gerufen. Der Anstoß zur Entwicklung der Initiative fand auf der ersten World's Large Rivers Konferenz statt, welche 2011 in Wien mit über 450 TeilnehmerInnen aus 73 Ländern veranstaltet wurde. Die Konferenz wurde von der UNESCO, der IAHR (International Association for Hydro-Environment Engineering and Research), der IAHS (International Association of Hydrological Sciences), WASER (World Association for Sedimentation and Erosion Research) und der IAG (International Association of Geomorphologists) unterstützt und gefördert. Die Delegierten und wissenschaftlichen ExpertInnen sprachen eine Empfehlung aus, wonach sich eine gemeinschaftliche, multidisziplinäre und internationale Initiative der wissenschaftlichen Bearbeitung und Begutachtung des Status und der möglichen zukünftigen Entwicklung der großen Flüsse der Welt widmen sollte. Um dieses Vorhaben auf UN-Ebene $\mathrm{zu}$ starten wurde die WLRI 2012 während der 20. Sitzung des Intergovernmental Councils des International Hydrological Programme der UNESCO (UNESCO-IHP) präsentiert. Die Idee der Initiative wurde unter den Delegierten diskutiert und fand großen Zuspruch. Die World's Large Rivers Initiative wurde in Folge während der 21. Sitzung des Intergovernmental Council (IGC) im Juni 2014 mit Auflagen angenommen. Im Juni 2018 wurde die WLRI dann final vom IGC beschlossen und ist nun offiziell Teil des UNESCOIHP-Arbeitsprogramms. Damit stellt die WLRI das erste von Österreich koordinierte UNESCO-IHP-Programm dar.

2014 wurde auch der UNESCO-Lehrstuhl „Integrated River Research and 


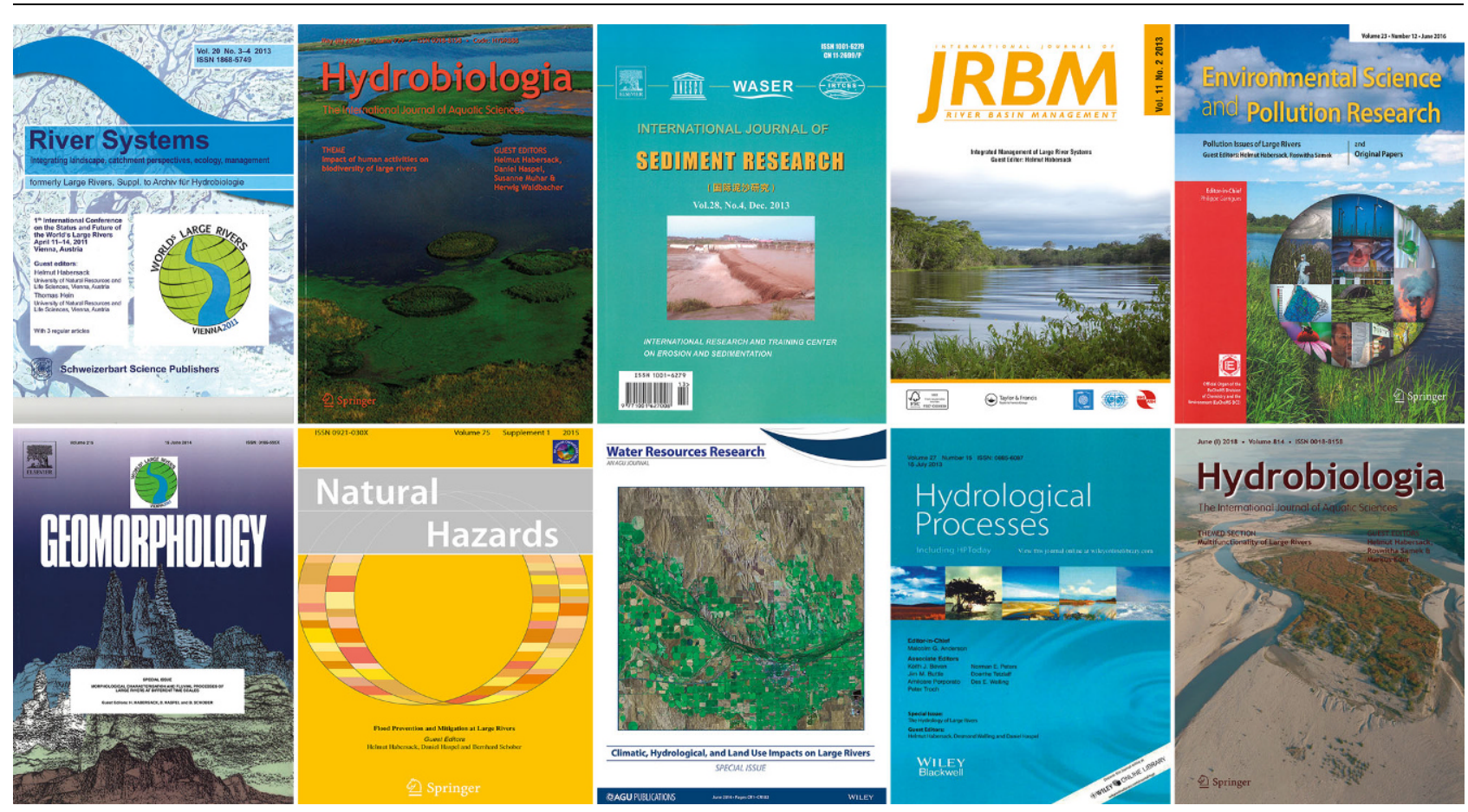

Abb. 1 10Special Issues als bisheriger wissenschaftlicher Output der WLRI: Hydrobiologia, 814, 1-246: Multifunctionality of Large Rivers. Environmental Science and Pollution Research, 23, 11393-12490: Water quality issues and management of large rivers. Natural Hazards, 75, 1-105 Flood prevention and mitigation at large rivers. Water Resources Research, 50, 3641-4544: Large Rivers in the Anthropocene: Insights and tools for understanding climatic, land use, and reservoir influences. Hydrobiologia, 729, 1-259: Impact of human activities on biodiversity of large rivers. Geomorphology, 215, 1-106: Morphological characterization and fluvial processes of large rivers at different time scales. River Systems, 20, 145-287: Integrating landscape, catchment perspectives, ecology, management. International Journal of River Basin Management, 11, 137-236: Integrated management of large river systems. Hydrological Processes, 27, 2103-2224: The Hydrology of Large Rivers. International Journal of Sediment Research, 28, 431-598: Sediment loads and processes in large rivers

Management“ an der Universität für Bodenkultur Wien gegründet (Inhaber ist Helmut Habersack). Der Lehrstuhl sieht die Koordination der Aktivitäten der World's Large Rivers Initiative als eine seiner Hauptaufgaben an. Damit ist Österreich auch Teil der sogenannten UNESCO Water Family, die neben den „Water Chairs“ auch die „Water Centres" umfasst.

Als rein wissenschaftliche Initiative zielt die WLRI darauf ab, einen globalen Bericht zum Status und der möglichen zukünftigen Entwicklung der großen Flüsse der Welt zu verfassen. Weitere Ziele sind das Training von ExpertInnen im Wassersektor, die Entwicklung eines internationalen ForschungsAktionsplans, die gezielte Vernetzung mit themenverwandten Organisationen und die Abhaltung von internationalen World's Large Rivers Konferenzen alle drei Jahre. In Phase I des Projekts wird die entwickelte Methodik an drei Flüssen getestet. Diese Flüsse sind die Donau, der Niger und der Mekong. In der darauffolgenden Phase II ist eine
Bearbeitung von bis zu 300 Flüssen unter Einbeziehung der Resultate von Phase I geplant.

$\mathrm{Zu}$ den Outputs der WLRI zählen auch die drei bisher organisierten World's Large Rivers Konferenzen. Nach Wien 2011 konnten weitere WLR-Konferenzen an diesen Erfolg anknüpfen und fanden 2014 in Manaus, Brasilien und 2017 in Neu-Delhi, Indien statt. Die Beiträge zu diesen Konferenzen führten zur Publikation von zehn Special Issues (Abb. 1) mit 143 Artikeln und 554 Autoren in internationalen SCIZeitschriften (ed. vom UNESCO Chair gemeinsam mit Co-Editoren). Weitere Special Issues sind derzeit in Ausarbeitung. Die nächste Konferenz ist für 2020 geplant und wird in Moskau, Russland stattfinden.

Besondere Aufmerksamkeit widmet die World's Large Rivers Initiative dem Sedimentmanagement im Zusammenhang mit Wasserkraftwerken. Die Speicherkapazität von Wasserkraftwerken auf globaler Ebene reduziert sich durch Verlandung derzeit schneller, als neue
Stauräume gebaut werden. Dies führt zu einem globalen Rückgang der Speicherkapazität, welcher sich auch in Zukunft fortzusetzen droht (Abb. 2), nach Annandale et al. 2018). Rechnet man die globale Speicherkapazität auf eine Pro-Kopf-Basis um, so begann die Trendwende bereits um 1980. Heute ist die Speicherkapazität pro Person wieder ähnlich dem Wert von 1960. Der Transport von Sedimenten ist ein natürlicher Vorgang, wird aber durch menschliche Eingriffe maßgeblich beeinflusst. Wasserkraftwerke tragen $\mathrm{zu}$ einer Unterbrechung des Sedimenttransports bei, können aber durch entsprechendes Design und Management deutlich verbessert werden. In enger Kooperation mit der WLRI wird derzeit ein globales Projekt entwickelt, um dem weltweiten Stauraumverlust entgegenzuwirken. Ziel ist es, ein nachhaltiges Sedimentmanagement an bestehenden und geplanten Wasserkraftwerken $\mathrm{zu}$ erreichen. Fallbeispiele aus mehreren Ländern werden untersucht, um sowohl positive als auch negative 


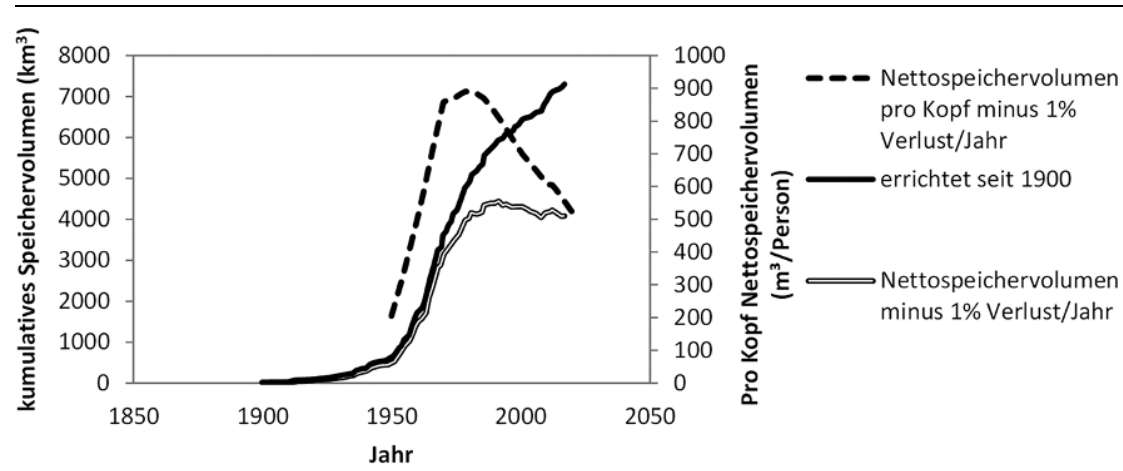

Abb. 2 Globale Speicherkapazität, Entwicklung des Stauraumvolumens seit 1900, Netto-Stauraumverlust durch Verlandung, Speicherkapazität pro Person (nach Annandale et al. 2018)

Praktiken zu dokumentieren. Ein technisches Toolkit bestehend aus einem Handbuch mit begleitender Software soll das Sedimentmanagement an Wasserkraftwerken unterstützen oder eine Verbesserung ermöglichen.

Unterstützend werden auch Richtlinien für politische Entscheidungsträger entwickelt, um Maßnahmen auf politischer, wirtschaftlicher, rechtlicher und regulatorischer Ebene zu identifizieren, die ein erfolgreiches Sedimentmanagement ermöglichen. Schlussendlich sind auch Aktivitäten im Bereich Capacity Building geplant, die unter anderem Workshops, Seminare, Konferenzen und Webinars umfassen.

Die WLRI sieht sich als eine „cross cutting“-Initiative, die sich „horizontal“ mit den „vertikalen“ thematischen Programmen vernetzt und die thematischen Ergebnisse auf konkrete Flüsse "projiziert“. Hervorzuheben ist dabei die International Sediment Initiative (ISI). Die ISI wurde 2002 eingerichtet, um auf globaler Ebene den Sedimenttransport ins Meer, in Seen oder Speicher zu analysieren und einen gesamtheitlichen Ansatz zur Verbesserung der Oberflächengewässer zu schaffen, unter enger Vernetzung von Wissenschaft, Management und Politik. Die Aktivitäten umfassen die Entwicklung einer Metadatenbank zum Sedimenttransport, die Erarbeitung von Fallbeispielen, die Definition sedimentrelevanter Aktivitäten und die Unterstützung von wissenschaftlichem Austausch, Bildung und Aufbau von Know-how, einschließlich Informationsmaterial und Kooperation mit anderen UNESCO-Programmen. Im Bereich Sedimentforschung erfolgt - über den UNESCO Chair als Mitglied von ISI und Koordinator von WLRI - eine enge Abstimmung.

\subsection{IAHR, WASER und weitere globale Forschungsgesellschaften}

Sedimenttransport wird in sehr vielen internationalen Wissenschaftsgesellschaften behandelt. Diese sind thematisch organisiert. Als Beispiele können folgende Gesellschaften genannt werden, eine gesamthafte Darstellung würde den Rahmen dieses Artikels sprengen:

- International Association for Hydro-Environment Engineering and Research (IAHR, https://www.iahr. $\operatorname{org} /)$ :

Die 1935 gegründete und ursprünglich auf Hydraulik fokussierte Gesellschaft behandelt Fluss- und Meereshydraulik bis hin zu Ökohydraulik, Hydroinformatik und hydraulische Maschinen. Der Sedimenttransport ist zunehmend vertreten. So wird es im 38. Weltkongress 2019 in Panama ein eigenes Thema „River and Sediment Management" geben. Der 40. Weltkongress findet 2023, koordiniert von der BOKU erstmals in Wien, statt und wird gemeinsam mit dem der 5. International Conference on the Status and Future of the World's Large Rivers (sh oben) abgehalten.

- World Association for Sedimentation and Erosion Research (WASER, http://www.waser.cn/waser/index. htm):

Das „International Research and Training Center on Erosion and Sedimentation (IRTCES)“, welches 1984 mit Sitz in Peking/China, gegründet wurde, koordiniert WASER. WASER konzentriert sich ausschließlich auf alle Aspekte der Sedimentforschung und deren interdisziplinärer Natur. Hervorzuheben ist die zweijährige Konferenzserie on River Sedimentation, deren 14. Ausgabe
2019 in Chengdu in China stattfindet (http://www.isrs2019.cn). Das Konferenzmotto "Integrated Sediment Management in Rivers and Coasts" umfasst die Themen „Sediment Yield and Erosion Processes, Sediment Transport, Sedimentation in Estuarine and Coastal Areas, Reservoir Sedimentation, Environmental and Ecological Sediment, Fluvial Processes and Geomorphology, Sediment Related to Disasters, Modeling \& Measurement Techniques, Integrated Sediment Management". Dies zeigt den umfassenden Ansatz. Wichtig zu erwähnen ist das SCI International Journal on Sediment Research (https://www.journals.elsevier.com/ international-journal-of-sedimentresearch), welches von WASER gemeinsam mit IRTCES herausgegeben wird. Der Erstautor dieses Artikels fungiert als Vizepräsident und associate editor.

- Weiters findet sich der Sedimenttransport als wichtiges Thema bei der International Association of Hydrological Sciences (IAHS, https:// iahs.info), wo die „International Commission on Continental Erosion (ICCE)" schon seit 30 Jahren sedimentrelevante Konferenzen abhält und in der bekannten „Red Book Series“ die entsprechenden Beiträge publiziert. Beispielsweise fand „The International Symposium Climate Change Impacts on Sediment Dynamics: Measurement, Modelling and Management" gemeinsam mit dem „The Second International Youth Forum on Soil and Water Conservation“ im August 2018 in Moskau statt. Der Erstautor hielt eine Keynote Lecture zum Thema „River Basin: Sediment Transport, Reservoir Sedimentation and Management".

- Die International Association of Geomorphologists (http://www.geo morph.org) zielt auf die Entwicklung der Geomorphologie als Wissenschaftsdisziplin und wurde 1989 gegründet, hält regelmäßige Konferenzen und workshops ab und unterstützt die World's Large Rivers Konferenzen (sh oben). Da ein Großteil der Erdoberfläche aus Sedimentgesteinen besteht und Flüsse die Leitlinien der Erosion darstellen, ist die Sedimentforschung für die Geomorphologie essenziell.

- Im Bereich der Geophysik/Geowissenschaften sind die American Geophysical Union (AGU, https://sites. 
agu.org) und European Geosciences Union (EGU, https://www.egu.eu) $\mathrm{zu}$ nennen. In den jeweiligen Konferenzen findet sich das Thema Sedimente (z. B. „Erosion, Sedimentation \& River Processes: Measurement and Monitoring Approaches" bei der EGU 2019 in Wien). Wichtig sind betreffend Sedimentforschung die AGU-SCI-Zeitschriften Water Resources Research und Geophysical Research Letters und EGU-SCI-Zeitschriften wie Hydrology and Earth System Sciences (HESS) oder Natural Hazards and Earth System Sciences (NHESS). Dabei haben bei der EGU in Wien die Sessions „Geomorphology“, „Hydrological Sciences“, „Natural Hazards“, „Stratigraphy, Sedimentology \& Palaeontology“ oder „Soil System Sciences“ häufig einen Sedimentbezug.

- Die International Society of River Science veranstaltet zweijährliche Konferenzen, 2019 an der BOKU, koordiniert vom IHG (http:// isrs2019.info/cms/index.php/home235.html) und in Partnerschaft mit dem BOKU-UNESCO-Lehrstuhl. In verschiedenen Sessions wird das Thema Sedimenttransport relevant, vor allem künftig in Bezug auf die aquatische Ökologie, den ökologischen Zustand und die Ökosystemleistungen (sh unten), wobei zu diesen Themen auch auf die International Society of Limnology (SIL, https://limnology.org) hingewiesen werden kann, die alle drei Jahre eine internationale Konferenz abhält (2020 die 35. Konferenz).

- Schließlich wird noch die International Commission On Large Dams (ICOLD, https://www.icold-cigb.org) angeführt, die 2018 in Wien einen erfolgreichen Weltkongress abhielt (geleitet von der TU Graz) und wo z. B. die Sessions „New Approaches to Sediment Flushing and Routing Methods Including Density Current Venting, Sluicing and Bypassing“ oder "Sedimentation of Reservoirs" stattfanden.

- Die International Hydropower Association (IHA, https://www.hydro power.org) hat 2017 den Hydropower Sediment Management Knowledge Hub initiiert, wo auch die Weltbank mitinvolviert ist.

\section{SedNet - eine europäische Initiative}

Das European Sediment Network (SedNet, https://sednet.org) wurde aus einem thematischen Netzwerk, gefördert durch die Europäische Kommission, gestartet. SedNet führt ExpertInnen aus Wissenschaft, Verwaltung und Industrie zusammen. SedNet interagiert mit verschiedenen Netzwerken in Europa, von der nationalen bis zur internationalen Ebene. Es zielt ab auf die Einbeziehung der Sedimentthemen und des Sedimentwissens in europäische Strategien, um die Umweltziele zu erreichen und auf die Entwicklung neuer Tools zur Unterstützung des Sedimentmanagements.

Basierend auf ursprünglich vier Büchern hat sich SedNet seit 2005 kontinuierlich weiterentwickelt, mit Partnern aus der Wissenschaft, Verwaltung und politiknahen EntscheidungsträgerInnen. SedNet hat seinen Fokus ebenfalls erweitert und deckt derzeit alle Bereiche des Sedimenttransports ab, von den Flüssen bis zum Meer. Die 11. Konferenz findet 2019 in Dubrovnik statt („Sediment as a Dynamic Natural Resource from Catchment to Open Sea“). Drei Runde Tische brachten internationale ExpertInnen aus mehreren europäischen Flusseinzugsgebieten zusammen (2006, 2009 und 2016). SedNet produzierte drei Position Papers/Policy Briefs: „Contaminated Sediments in European River Basins“ (2004), „Moving Sediment Management Forward - The Four SedNet Key Messages“ (2014) und; „Effective River Basin Management Needs to Include Sediment" (2017), woraus nachstehend einige Punkte zusammengefasst sind. SedNet war ein wesentlicher Teilnehmer der Common Implementation Strategy (CIS) der Wasserrahmenrichtlinie, wirkt in Aktion 5 der EU-Biodiversitätsstrategie mit. SedNet trug zu einem Trainingskurs betreffend nachhaltiges Sedimentmanagement im Savaeinzugsgebiet bei.

Auf dieser Grundlage erarbeitete SedNet vier Kernbotschaften betreffend Sedimentmanagement (sh. https:// sednet.org/):

1. Sedimentquantität und Hydromorphologie:

Sedimenttransport ist eine vitale Komponente des natürlichen hydromorphologischen Regimes. Management der meisten europäischen Flüsse bedeutet, dass die natürlichen Sedimenttransportprozesse substan- ziell verändert wurden, teilweise mit dramatischen Konsequenzen für die Stabilität der Flüsse und Küsten. Die Wasserrahmenrichtlinie zielt z. B. auf die Erreichung eines guten ökologischen Zustands ab, wobei die Hydromorphologie und damit verbunden der Sedimenttransport entscheidend für die Zielerreichung sind. Das erfordert eine sorgfältige Risikoabschätzung, bevor Maßnahmen ergriffen werden.

2. Sedimentqualität und Remobilisierung:

Kontaminierte Sedimente können negative Auswirkungen auf die Menschen, die Umwelt und die Wirtschaft haben. Da die Sedimente durch Flusssysteme transportiert werden, treten derartige Effekte nicht nur lokal, sondern noch weit entfernt von den Quellen der Kontamination auf. Sanierungs- und Schutzmaßnahmen müssen daher in Flussgebietsbewirtschaftungspläne integriert werden. Um ein Management zu ermöglichen, wird ein besseres Verständnis der zugrunde liegenden Prozesse der Remobilisierung, des Phasentransfers, der Verfügbarkeit der Kontaminierung und des Transports besonders unter Extrembedingungen - benötigt. Handlungsbedarf besteht nicht nur in der Kontrolle der Quellen, sondern flussab in Zusammenhang mit Extremereignissen und auch mit dem Klimawandel.

3. Sediment und Fließgewässerökologie:

Sedimente besitzen eine fundamentale Bedeutung für aquatische Ökosysteme. Die Erreichung eines nachhaltigen Ausgleichs zwischen der Entwicklung der Wasserstraßen, Wasserkraft und Ökologie hängt von einem konstruktiven Dialog zwischen den verschiedenen Stakeholdern, einer besseren Koordination und einem effektiveren grenzüberschreitenden Management ab.

4. Management von Baggermaterial: Statt Baggermaterial als Abfall zu sehen, sollten Sedimente als Ressource verstanden werden. Dabei ist die Änderung der Perzeption von Baggermaterial wichtig. Die EU-Unterstützung ist dabei wichtig, um ein nachhaltiges Management von Baggermaterial innerhalb der bestehenden gesetzlichen Rahmenbedingungen zu ermöglichen. 
Die vier Kernbotschaften können folgendermaßen zusammengefasst werden: Sedimente sind ein integraler Bestandteil der Natur und aquatischer Systeme; sie sind eine wichtige Ressource, die Schutz und gezieltes Management erfordert.

Daraus folgt, dass eine effektive Flussgebietsbewirtbewirtschaftung die Einbeziehung von Sedimenten benötigt. SedNet veröffentlicht „Policy Briefs“, z.B. „Effective River Basin Management Needs to Include Sediment“, die der Europäischen Union das Thema Sedimente näherbringen:

Gemäß SedNet ist es klar und wissenschaftlich bewiesen, dass ein guter ökologischer Zustand nur mit einer ausgeglichenen Sedimentbilanz und guter Sedimentqualität erreicht werden kann. Aber hydromorphologische Veränderungen infolge Schifffahrt, Wasserkraftnutzung oder Hochwasserschutz und Kontamination infolge punktueller oder diffuser Quellen werden in den europäischen Flussgebietsbewirtschaftungsplänen als die zwei wichtigsten Gründe angeführt, warum Wasserkörper nicht den guten Zustand oder das gute Potenzial erreichen, wie es von der Wasserrahmenrichtlinie gefordert wird (SedNet 2017).

Baggerungen sind notwendig, um Häfen, schiffbare Wasserstraßen, Speicher für Trinkwasserversorgung oder Stromproduktion zu erhalten. Ein unterbrochenes Sedimentregime führt aber zur Degradierung der Flussmorphologie und als Folge zu einer Erhöhung des Hochwasserrisikos. Anlandungen in Überflutungsflächen können zu einer Reduktion der Hochwasserrückhaltefähigkeit führen. Die Sicherstellung einer ausreichenden Sedimentversorgung unterstützt daher die Ziele der EU-Hochwasserrichtlinie (SedNet 2017).

Die Relevanz der Sedimente zur Erreichung der grundlegenden Managementziele in Flussgebieten ist offensichtlich. Aber die hohe Komplexität verhindert die volle Integration der Sedimentthemen in das Flussgebietsmanagement.

SedNet bietet an, die Integration der Sedimente in der Flussgebietsbewirtschaftung durch Erfahrungsaustausch zu unterstützen:

- Verständnis der Sedimenttransportprozesse und Handlungsweisen auf strategischer und lokaler Ebene,
- Entwicklung und Implementierung von guten Sedimentmanagementkonzepten in der Praxis,

- Entwicklung und Förderung von guten praktischen Sedimentmanagementmaßnahmen,

- Identifikation und Evaluierung von Maßnahmen zur Sicherstellung, dass neue wasserbauliche Maßnahmen und andere Entwicklungen das Sedimentregime nicht negativ beeinflussen.

Es gibt eine Arbeitsgruppe „Sediments in Circular Economy“, und 2018 wurde innerhalb von SedNet die Gruppe „Sedimentquantität" gegründet, die vom Erstautor dieses Artikels gemeinsam mit einem holländischen Kollegen von Deltares geleitet wird. Derzeit wird dort u.a. an einem Grundlagenpapier „Managing Sediment Fluxes Linking Mountains, Rivers and the Sea" gearbeitet.

\section{Regionale internationale Aktivitäten betreffend Sedimente im Alpenraum}

\subsection{SedAlp}

Sediment Management in Alpine Basins: Integrating Sediment Continuum, Risk Mitigation and Hydropower (SedAlp, www.sedalp.eu/) ist ein Interreg "Alpine Space“ Projekt und fokussiert auf das integrative Management des Sedimenttransports in alpinen Einzugsgebieten. Es ist auf eine effektive Reduktion der sedimentbezogenen Risiken, die Verbesserung der aquatischen Ökosysteme und die Reduktion des Einflusses der Wasserkraftanlagen ausgerichtet.

Als „Key Outputs“ ergaben sich folgende Punkte (Skolaut et al. 2015):

\section{Sedimentproduktion}

- Die automatische Ermittlung von aktiven Erosionsbereichen an Hängen basierend auf Fernerkundungsmethoden stellt eine effiziente Vorgangsweise dar und terrestrische und airborne LiDAR-Daten stellen zeitintegrierte Erosionsraten an Hängen als Input für die Gerinne zur Verfügung.

- Die Ergebnisse zeigten, dass punktuelle Hot Spots der Erosion in den $\mathrm{Al}$ pen von kritischer Bedeutung für die Analyse des Sedimenteintrages in alpine Flüsse sind, welche sich bei Sedimentdefizit über $10 \mathrm{~cm} / J a h r$ eintiefen können.

\section{Sedimenttransport/Transfer}

- Neue und öffentlich verfügbare Datensätze betreffend Sedimente (Schwebstoffe, Geschiebe und Muren) und Wildholztransport stehen zur Verfügung.

- Die Messmethoden für den Sedimenttransport konnten im Alpenraum standardisiert werden.

- Die raum-zeitliche Variabilität des Sediment- und Wildholztransports wurde nachgewiesen.

- Möglichkeiten und Herausforderungen zur Verbesserung von Sedimentund Holztransportformeln wurden diskutiert und diese wurden anhand von Felddaten validiert.

Sedimentdeposition/Remobilisierung

- Ergebnisse der Evaluierung von Rückbauprojekten zeigten, dass Daten zum historischen und derzeitigen Sedimenttransport eine Grundvoraussetzung darstellen und eine einzugsgebietsweise Analyse des Feststoffhaushalts essenziell ist.

\section{Integratives Sedimentmanagement}

- Stärkung der historischen Analyse alpiner Einzugsgebiete durch Kombination von historischen Aufnahmen mit hochauflösenden digitalen Geländemodellen (DGM) abgeleitet von Laserscans.

- Die „Fluvial Corridor Toolbox“ welche im Projekt entwickelt wurde erlaubt die Extrahierung von verschiedenen Fließgewässermerkmalen.

- GIS-basierte tools wurden entwickelt um die räumlichen Muster der Konnektivität basierend auf einem räumlich verteilten Index über hochauflösende DGM zu berechnen.

- Ein Leitfaden für die Planung von effizienten Wildbachsperren mit geringer Auswirkung auf die Sedimentkontinuität beschreibt die Möglichkeiten und die Nutzung von mathematischen Modellierungen und physikalischen Experimenten.

- Ein Leitfaden für die Planung von optimierten Wasserkraftanlagen zielt auf die Verbesserung des Sedimentkontinuums zwischen den Einzugsgebieten und den flussabwärtigen Strecken. Managementpläne sollten Speicher- und Dammcharakteristik, morphologische und hydraulische Parameter, ökologische Auswirkungen, geologische Randbedingungen, Erosions- und Sedimentationsbereiche, Sedimentanalyse und Remobilisierungsmaßnahmen sowie Verbes- 


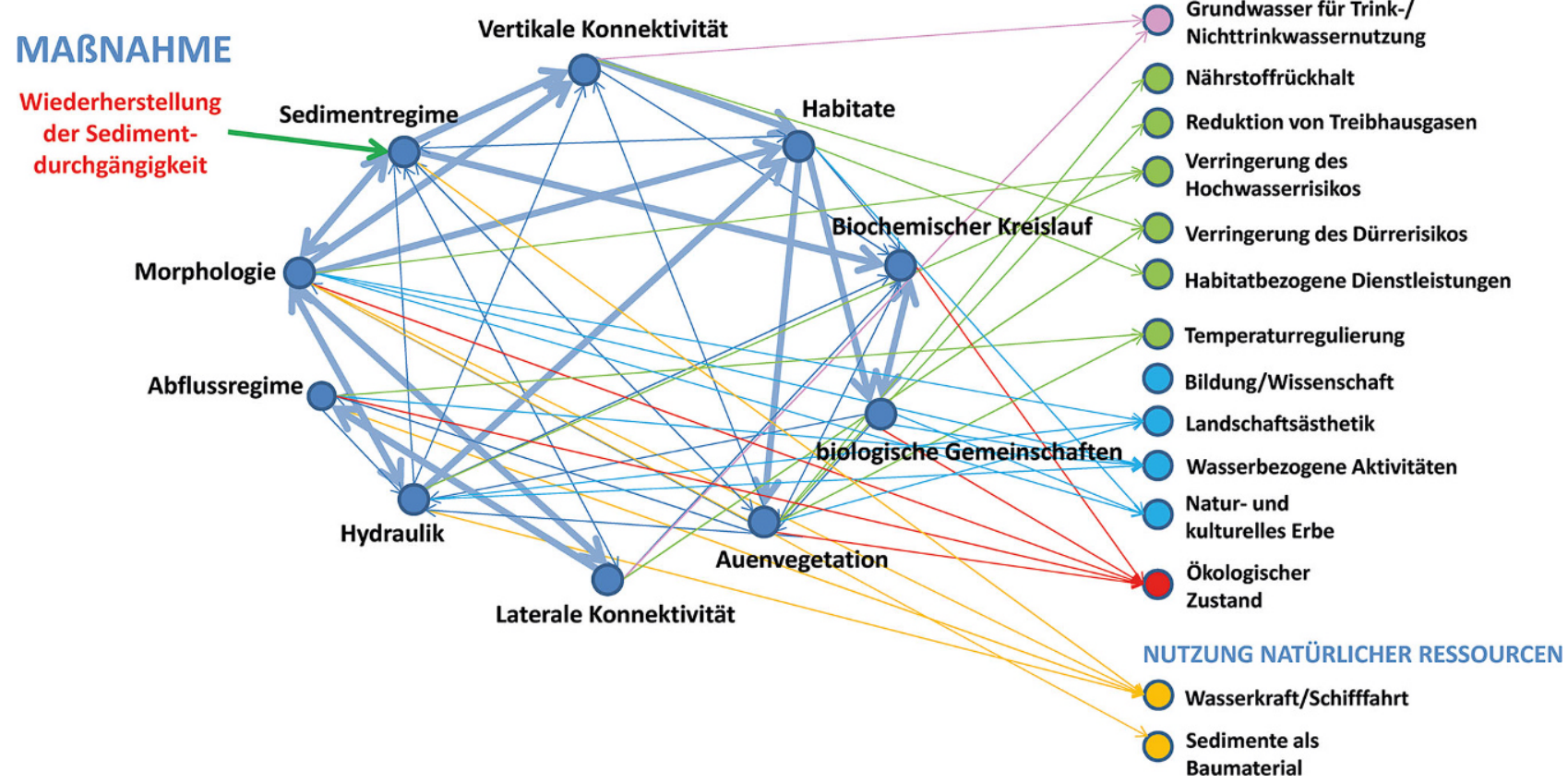

Abb. 3 Rahmenwerk zur Berücksichtigung von Ökosystemdienstleistungen in Planungs- und Managementaktivitäten mit Einfluss auf hydromorphologische Prozesse. Die Maßnahme (hier: Wiederherstellung der Sedimentdurchgängigkeit) greift in das funktionale Netzwerk der Flussfunktionen ein und wirkt sich auf die Bereitstellung von Ökosystemdienstleistungen für den Menschen aus. Den Verknüpfungen sind Werkzeuge zugeordnet, welche den Funktionsablauf und die Auswirkung auf die Ökosystemdienstleistungen berechnen lassen (basierend auf HyMoCARES 2019)

serungsmaßnahmen für Flusssysteme (Schutz aquatischer Ökosysteme etc.) umfassen.

Weiters wurden Empfehlungen für die Praxis, Politik und Forschung abgeleitet (Aigner et al. 2014).

Eine Weiterentwicklung auf Basis des Projektes SedAlp stellt das „Hydromorphological Evaluation Tool (HYMET)“ dar, welches ein hierarchisches Prozedere für die Bewertung des Sedimentinputs und -transfers im Flusssystem als Voraussetzung für eine nachhaltige Morphodynamik in Flüssen vorstellt (Klösch und Habersack 2017).

\subsection{HyMOCARES}

Der Zustand der Fließgewässer und deren Ökosysteme sind für das Wohlbefinden und die Wohlfahrt des Menschen von großer Bedeutung. Dass der Mensch seinen Nutzen aus den Ökosystemen der Flüsse zieht, wurde mit deren Reduktion bzw. deren Verlust nach zum Teil übermäßigen und unkoordinierten Eingriffen deutlich. Einige Studien be- fassten sich bereits damit, die Relevanz der Ökosysteme für den Menschen an konkreten „Ökosystemdienstleistungen“ festzumachen. Das von der Europäischen Union im Rahmen des Interreg Alpenraumprogramms geförderte Projekt HydroMorphological Assessment and Management at Basin Scale for the Conservation of Alpine Rivers and Related Ecosystem Services (HyMoCARES, https://www.alpine-space. eu/projects/hymocares/en/home) zielt nun darauf ab, von alpinen Fließgewässern bereitgestellte Ökosystemdienstleistungen in Planungs- und Managementaktivitäten $\mathrm{zu}$ integrieren. In diesem Projekt arbeiten 13 Partner aus den Alpenländern, darunter das Institut für Wasserbau, Hydraulik und Fließgewässerforschung der Universität für Bodenkultur Wien (Lead Partner: Agentur für Bevölkerungsschutz der Autonomen Provinz Bozen, Südtirol).

Insbesondere die Sedimentdurchgängigkeit und die damit verbundenen hydromorphologischen Prozesse haben eine Schlüsselrolle in der Erhaltung von Habitaten und stehen in
Zusammenhang mit strategischen gesellschaftlichen Herausforderungen wie Hochwasserschutz, Energieproduktion, Artenschutz und Erholungsfunktion.

Das laufende Projekt HyMoCARES klärt die Zusammenhänge zwischen Nutzungen, hydromorphologischen Prozessen und die Verfügbarkeit von Ökosystemdienstleistungen und legt der Bevölkerung die Ökosystemdienstleistungen transparent dar. Konfliktlösungen und die Suche nach kosteneffizienten Maßnahmen sollen dadurch erleichtert werden. Das Projekt erarbeitet einen bisher nicht verfügbaren konzeptionellen Rahmen sowie einsatzfähige Werkzeuge mit neuen Methoden, um Ökosystemdienstleistungen in die Planung und in das Management der alpinen Flussgebiete zu integrieren. Die Praxistauglichkeit wird durch die Einbindung eines transnationalen Netzwerks aus EntscheidungsträgerInnen, BeamtInnen, PraktikerInnen mit Planungs- und Managementaufgaben sowie durch beispielhafte Anwendung in Fallstudien (in Österreich sind dies 


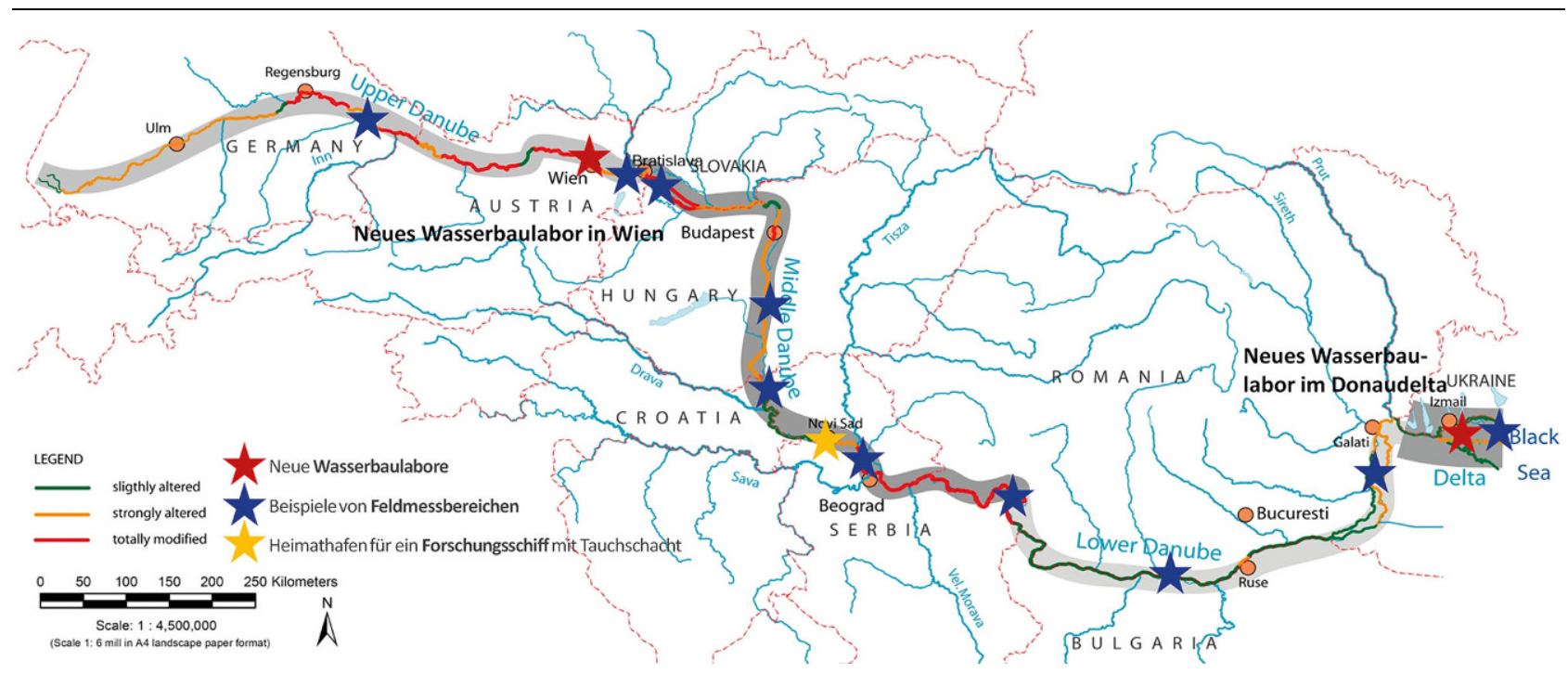

Abb. 4 Standorte für die Umsetzung von Forschungsinfrastrukturen an der Donau

Flussabschnitte an der Drau, Mur und Salzach), gewährleistet.

Der Brückenschlag zwischen flussbaulichen Maßnahmen und Ökosystemdienstleistungen setzt eine Kenntnis der in einem Fluss ablaufenden Prozesse voraus, die in HyMoCARES in Flussfunktionen (z.B. Hydraulik, Morphologie, etc.) zusammengefasst werden. Jede wasserbauliche Maßnahme bewirkt nun Veränderungen dieser Funktionen, die sich schlussendlich auf die Ökosystemdienstleistungen auswirken. Den Verknüpfungen in diesem Rahmenwerk werden existierende und neu entwickelte Werkzeuge zugeteilt, die zur Berechnung des Einflusses unterschiedlicher Maßnahmen auf die Ökosystemdienstleistungen empfohlen werden. In Abb. 3 ist die Funktionsweise des Rahmenwerks für die Sedimenteinbringung als ein Maßnahmenbeispiel dargestellt. Das Rahmenwerk und die zugeordneten Werkzeuge können gegen Ende des Projekts online abgefragt werden.

\section{Internationale sedimentrelevante Aktivitäten im Donau-, Rhein- und Elbeeinzugsgebiet}

\subsection{DREAM}

Wasserrahmenrichtlinie und Danube River Basin Management Plan fordern die Integration von Nutzung und nachhaltigem Schutz der Donau. Dies erfordert neben Grundlagenforschung und angewandter Forschung Lösungen im Bereich der Monitoringstrategien, Modellierung und im Management.
Diese liefern geeignete Maßnahmen, um eine Win-Win-Situation zwischen wirtschaftlicher Nutzung und Schutz der Donau zu erreichen.

DREAM (Danube River REsearch and Managment) ist ein EUSDR PA7 Flagship Projekt, unter dessen Schirmherrschaft unter anderem Infrastruktur für eine Reihe von Forschungsthemen bereitgestellt werden soll.

Diese Themen sind in sechs Aktivitäten miteinander verbunden und decken mehrere Disziplinen ab, von der Grundlagenforschung über hochmoderne Wasserbaulabore und hochentwickelte numerische 3D-Modelle bis hin zur angewandten Forschung, die Felddaten bereitstellt, um hydrologische Extreme abzumildern oder Schäden zu verhindern und bestehende Situationen betreffend Fluss- und Sedimentregimes $\mathrm{zu}$ verbessern (siehe auch Abb. 4):

Akt. 1: Errichtung von zwei großen „Responsible River Modelling Centers/Wasserbaulabors" (bis $10 \mathrm{~m}^{3} / \mathrm{s}$ Labordurchfluss): eines an der Oberen/Mittleren Donau und eines im Unterlauf/Delta.

Akt. 2: Kooperation existierender Wasserbaulabors zur Verbesserung der technischen Kompetenz in allen Partnerländern.

Akt. 3: Entwicklung eines Clusters/ Netzwerks von Fließgewässersimulationsmodellen für den Donauraum.

Akt. 4: Einrichtung eines Netzwerks von Feldmessstationen ent- lang der Donau und ihrer Zubringer.

Akt. 5: Bau und Betrieb eines gemeinsamen Forschungsschiffs mit Tauchschacht für den Einsatz (auch für praktische wasserbauliche Fragestellungen wie Inspektion von Brückenpfeilern etc.) entlang der ganzen Donau.

Akt. 6: Bildung eines Netzwerks von bestehenden und erweiterten Forschungseinrichtungen betreffend Nutzung und Schutz der Fließgewässer über alle Donauländer.

Folgende Themen können in $\mathrm{Zu}$ sammenarbeit mit den Partnerländern und Institutionen, die die DREAM-Infrastruktur nutzen, untersucht werden:

- Grundlagenforschung zu Sedimenttransport, Strömungsbedingungen, Ökologie, Auswirkungen auf den Menschen und mögliche zukünftige Maßnahmen.

- Integriertes Hochwasserrisikomanagement (Dämme, mobiler Hochwasserschutz etc.).

- Flussbau, -rückbau (Schwerpunkt Sedimenttransport).

- Erneuerbare Energie (insbesondere Wasserkraft).

- Verkehr und Transport (Infrastruktur in der Wasserstraße Donau, Schifffahrt).

- Fließgewässerforschung.

Ein wichtiges Ziel des Projekts DREAM ist es, die Erforschung von hydrodynamischen, morphodynamischen und 


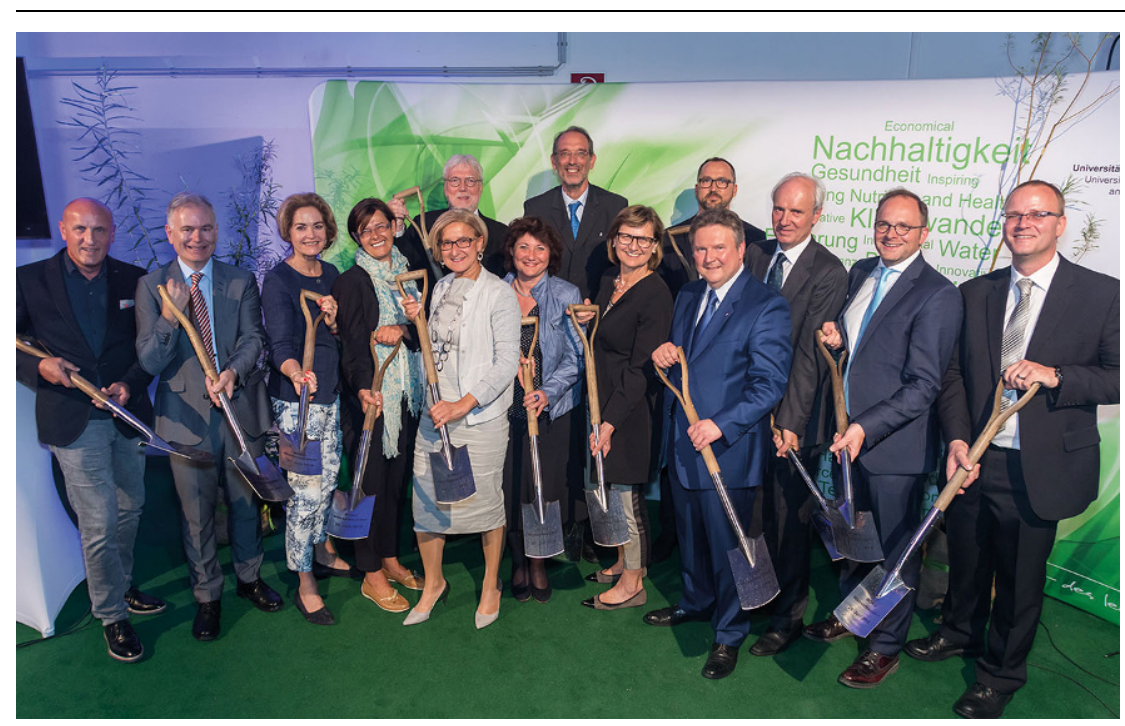

Abb. 5 Spatenstich BOKU-Wasserbaulabor im Juni 2018 als Teil des EUSDR Flagship Projektes DREAM (v. I.): Architekt DI Horst Reiner (ATP), Univ.-Prof. DI Dr. Helmut Habersack (BOKU, Projektleiter), ALin Dr. Ulrike Unterer (BMDW), Vizerektorin Mag.a Andrea Reithmayer (BOKU), NÖ Landeshauptfrau Mag. a Johanna Mikl-Leitner, Dir. EU DG Regio Erich Unterwurzacher, Mag.a Margit Harjung (BMVIT), Bundesminister Univ.Prof. Dr. Heinz Faßmann (BMBWF), SCin DI Maria Patek (BMNT), DI Hartwig Schindler (iC Consulenten), Wiens Bürgermeister Dr. Michael Ludwig, Rektor Univ.-Prof. DIDr. Hubert Hasenauer (BOKU), DI Maximilian Pammer (BIG), GF Mag. Jürgen Gruber (BOKU) (Foto: BOKU)

ökologischen Prozessen sowie des Sedimenttransports in verschiedenen Bereichen der Donau durch geeignete Wasserbaulabors $\mathrm{zu}$ ermöglichen, die einen einzigartigen Durchfluss (bis zu $10 \mathrm{~m}^{3} / \mathrm{s}$ ohne Pumpen) ermöglichen.

Auf der Grundlage eines verbesserten Prozessverständnisses, das von den physikalischen Modellen in den Labors abgeleitet wird, sollten computergestützte Simulationen verbessert werden, die zu Hybridmodellen führen. Ein weiteres Ziel ist es, gemeinsam vereinbarte Felduntersuchungsstandorte und -stationen entlang der Donau zu errichten, um physikalische und computerbasierte Modelle $\mathrm{zu}$ kalibrieren und $\mathrm{zu}$ validieren sowie fortschrittliche Verfahren für den Flussbau unter 1:1-Bedingungen $\mathrm{zu}$ entwickeln und $\mathrm{zu}$ testen.

Die Zusammenarbeit von Forschungseinrichtungen und -labors entlang der Donau soll den wissenschaftlichen Fortschritt verbessern und den Übergang von der Grundlagenforschung zum nachhaltigen Management und zur Wissensgesellschaft anregen.

DREAM führt $\mathrm{zu}$ einer grundlegenden Verbesserung der Forschungsinfrastruktur und der Zusammenarbeit zwischen Forschungseinrichtungen im Donauraum. Die neuen Wasserbaula- bors bieten in Kombination mit den bestehenden Labors eine einzigartige Möglichkeit für die Anwendung von physikalischen Modellen im großen Maßstab (bis 1:1), von der Grundlagenforschung bis zur angewandten Forschung. Gerade die großmaßstäblichen Modellversuche sind in der Sedimenttransportforschung sehr wichtig, da viele derzeit verwendete Formeln in der Praxis Limitationen aufzeigen und Skaleneffekte zu beobachten sind.

Im Juni 2018 wurden mit einem internationalen Event in Wien die drei grenzüberschreitenden Projekte „Danube River Research and Management in Slovakia and Austria, DREAM SK-AT (INTERREG V-A SK-AT)“, „Sedimentforschung und -management an der Donau II, SEDDON II (INTERREG VA HU-AT)“ und „Sedimente, Ökosystemdienstleistungen und Wechselwirkungen mit Hochwasser und Dürre in der AT-CZ-Grenzregion, SEDECO (INTERREG V-A AT-CZ)“ und ein Wiener EFRE-Projekt („DREAM RRMC VIENNA (Wasserbaulabor)“) gestartet. In diesem Rahmen fand auch der feierliche Spatenstich für das einzigartige Wasserbaulabor mit bis $\mathrm{zu} 10 \mathrm{~m}^{3} / \mathrm{s} \mathrm{La-}$ bordurchfluss ohne Pumpen in Wien als Teil von DREAM statt (s. Abb. 5).

\subsection{DanubeSediment}

Maßnahmen des Gewässermanagements wie auch des Sedimentmanagements sind vor allem dann wirksam, wenn sie im Zuge einer Gesamtstrategie und eines einzugsgebietsorientierten Ansatzes umgesetzt werden, dem auch die Wasserrahmenrichtlinie (WRRL) folgt.

Ein Projekt, das diesem ganzheitlichen Ansatz entspricht und wichtige Beiträge zum 3. Internationalen Bewirtschaftungsplan für die Donau (Danube River Basin Management Plan, DRBMP) und zum 2. Hochwasserrisikomanagementplan der Donau (Danube Flood Risk Management Plan, DFRMP) liefern soll, ist das von der EU im Rahmen des DTP-Programms geförderte Projekt „Danube Sediment Management Restoration of the Sediment Balance in the Danube River" (DanubeSediment). Im Rahmen dieses Projekts, in dem 15 Partner - darunter auch das Institut für Wasserbau, Hydraulik und Fließgewässerforschung der Universität für Bodenkultur Wien - aus neun Donauländern beteiligt sind, wird erstmals eine Sedimentbilanz für die gesamte Donau erstellt (Lead Partner: Budapest University of Technology and Economics). Diese soll, primär basierend auf bereits existierenden Daten, den Sedimenttransport der Donau quantifizieren und Bereiche mit Sedimentdefizit und -überschuss identifizieren.

Um die Flüsse besser nutzen zu können, wurden die Donau und ihre Nebenflüsse schrittweise für den Hochwasserschutz, die Schifffahrt und später für die Wasserkraft verändert. Die Auswirkungen dieser Entwicklungen auf den Fluss werden durch punktuelle und diffuse Verschmutzungen und Auswirkungen der Landnutzungsänderung, wie landwirtschaftliche Intensivierung und forstwirtschaftliche Entwicklung, noch verstärkt. Diese Entwicklung trifft nicht nur den Hauptstrom der Donau selbst, sondern wirkt sich auch auf deren Überflutungsflächen und Transportkapazitäten aus (Habersack et al. 2016). Als Folge der zahlreichen Eingriffe hat sich das Sedimentregime der Donau im letzten Jahrhundert drastisch verändert. Zwischen 1950 und 1980 wurden insgesamt 69 Stauseen im Einzugsgebiet der Donau gebaut (1998 kam das Kraftwerk Freudenau dazu), die ein Gesamtvolumen von rund $7,3 \times 10^{9} \mathrm{~m}^{3}$ aufweisen. Die Stauräume an der Donau selbst machen dabei rund $50 \%$ 
Änderung der mittleren jährlichen Schwebstofffracht im Längsverlauf der Donau (1986-2016) im Vergleich zum Zeitraum vor Errichtung der Wasserkraftwerke

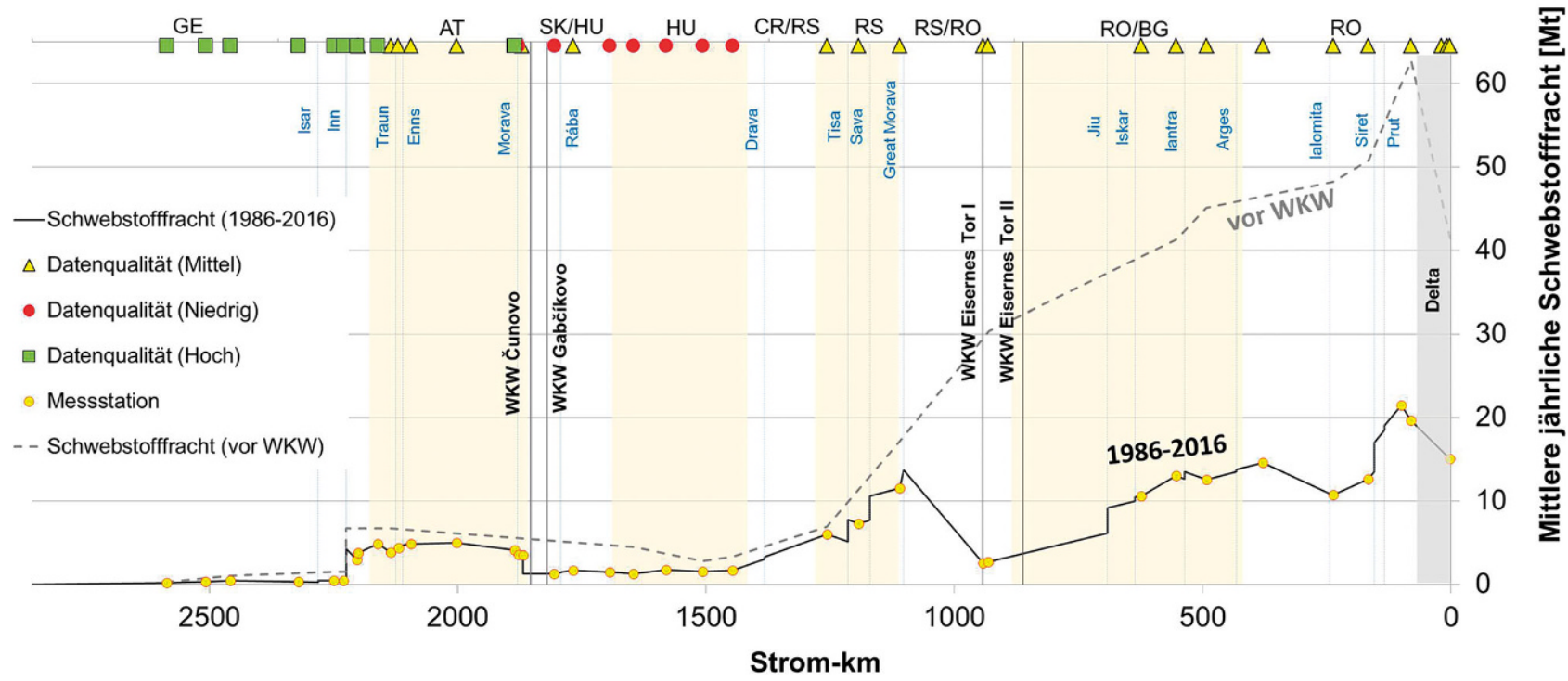

Abb. 6 Vergleich des Schwebstofftransports an der Donau vor Kraftwerkserrichtung (WKW) mit dem Zeitraum 1986-2016. (Modifiziert nach DanubeSediment WP32019)

des Gesamtvolumens aus (Dogterom 2001). In den Stauräumen der Kraftwerke kommt es aufgrund der verringerten Fließgeschwindigkeiten und Transportkapazitäten zu Ablagerung und folglich $\mathrm{zu}$ einem Überschuss an Sedimenten. Bei großen Hochwasserereignissen können die feinen Sedimente etwa in den Stauräumen der Oberen Donau remobilisiert werden, bei Ausuferungen zu Anlandungen in den Überflutungsflächen führen und damit zu großen Schäden im bebauten und bewirtschafteten Vorland beitragen (Habersack et al. 2015). Die Auswirkungen setzen sich bis zum Donaudelta fort: Heutzutage gelangt weniger als die Hälfte der ehemals transportierten Schwebstofffracht pro Jahr ins Delta bzw. Schwarze Meer (Abb. 6, DanubeSediment WP3, in Vorbereitung).

Die verbleibenden fünf freien Fließstrecken der Donau erfahren ein Sedimentdefizit durch die Unterbrechung des Sedimentkontinuums. Zusätzlich wurde die Gewässerbreite der Donau reduziert (Abb. 7) und durch die Laufverkürzung und Kanalisierung des $\mathrm{Ge}$ rinnes das Gefälle erhöht (aus DanubeSediment WP4, geleitet von VUVH SK, in Vorbereitung). Dies führt zusätzlich zu einer Erhöhung der Sedimenttransportkapazität des Flusses. Darüber hinaus wird durch die Kombination von eingeschränkter lateraler Erosion und
Verzweigung der seitliche Sedimenteintrag begrenzt.

Als Konsequenz von Sohlabsenkungen im Rahmen von Hochwasserschutzmaßnahmen oder Sedimentbaggerungen kann rückschreitende Erosion auftreten und der Transport stromab wird unterbunden bzw. reduziert, da sich in diesen Bereichen verstärkt Sediment ablagert. Diese Faktoren gemeinsam führen in den freien Fließstrecken zu einer Erosion der Donausohle in der Größenordnung von bis zu mehreren Zentimetern pro Jahr (Habersack et al. 2016). In weiterer Folge kommt es zu einer Absenkung des Grundwasserspiegels, Seitenarme werden abgetrennt, Flussstrukturen gehen verloren und die Habitatqualität verschlechtert sich, was den ökologischen Zustand wertvoller Überschwemmungsgebiete beeinträchtigt (Habersack et al. 2016). Die Auswirkungen setzen sich bis zur Küstenmorphologie fort: hier kann eine Küstenerosion am Schwarzen Meer von maximal 20 bis $25 \mathrm{~m}$ jährlich festgestellt werden (Stanica und Panin 2009). Im Donaudelta führt der Mangel an Sedimenten gemeinsam mit dem Abtrennen von Mäandern auch zur Sohlerosion der Hauptarme (Schwarz et al. 2008).

Im Projekt „DanubeSediment“ werden neben Auswirkungen auf den Sedimenthaushalt auch die wichtigsten Faktoren für die Veränderungen erho- ben und Maßnahmen zur Verbesserung des Sedimentkontinuums zusammengestellt. Auf die gewonnenen Erkenntnisse aufbauend werden Empfehlungen für ein gemeinsames, nachhaltiges Sedimentmanagement formuliert. In diesen Prozess sollen alle wichtigen Stakeholder etwa aus den Bereichen Wasserkraft, Binnenschifffahrt, Naturschutz und Hochwasserschutz, vor allem durch Teilnahme an Workshops, eingebunden werden. Die Ergebnisse und Empfehlungen sollen in einer „Danube Sediment Management Guidance" kurz zusammengefasst und in einem „Sediment Manual for Stakeholders" bezüglich der Bereiche Schifffahrt, Wasserkraft, Hochwasserschutz und Flussgebietsmanagement einschließlich Ökologie ausführlich diskutiert werden.

\subsection{Grenzüberschreitende Projekte im Donaueinzugsgebiet mit Ungarn, der Slowakei und Tschechien}

Die drei grenzüberschreitenden Projekte „Danube River Research and Management in Slovakia and Austria, DREAM SK-AT (INTERREG V-A SKAT)“, „Sedimentforschung und -management an der Donau II, SEDDON II (INTERREG V-A HU-AT)“ und „Sedimente, Ökosystemdienstleistungen und Wechselwirkungen mit Hochwasser und Dürre in der AT-CZ Grenzregi- 


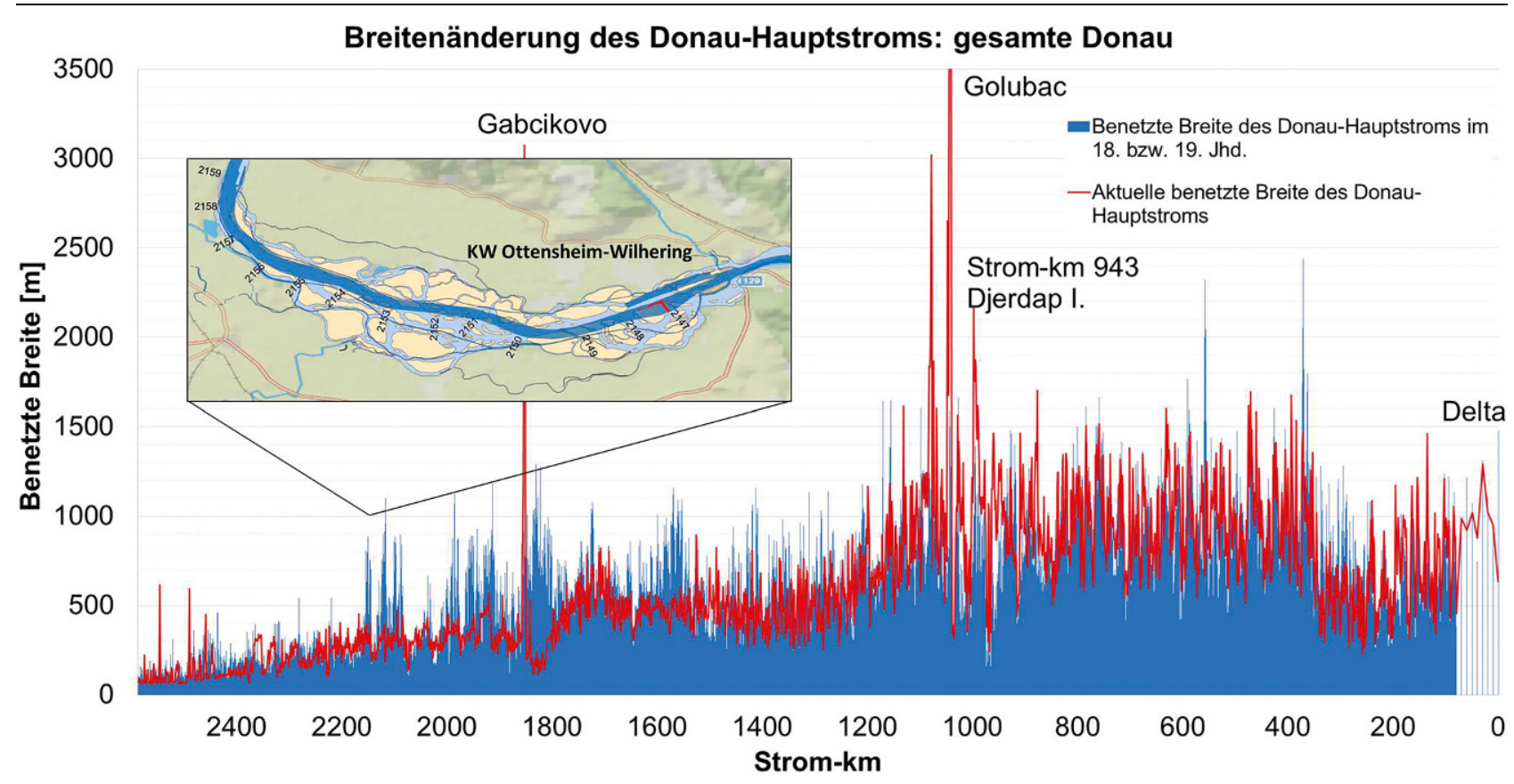

Abb. 7 Veränderung der Gewässerbreiten an der Oberen Donau zwischen historischem und aktuellem Zustand (modifiziert nach DanubeSediment WP4 2019)

on, SEDECO (INTERREG V-A AT-CZ)“ behandeln Sedimentfragen in bilateralen Kooperationen zwischen Österreich und der Slowakei, Ungarn und der Tschechischen Republik. In allen drei Projekten werden in den Nachbarländern Sedimentmonitoringstationen in Anlehnung an die in Österreich verwendete Methodik errichtet und Messungen durchgeführt. Mithilfe numerischer Simulationen und physikalischer Modellversuche erfolgen einerseits grundlegende Untersuchungen zum Prozessverständnis einschließlich des Einflusses von Skalen auf das Modellergebnis bis hin zur Entwicklung und Überprüfung von Maßnahmen zur Verbesserung der sedimentrelevanten Verhältnisse. Dabei reicht das Spektrum von Maßnahmen zur Verringerung der Sohleintiefungen bis zur Verbesserung des ökologischen Zustands und Optimierungen in Zusammenhang mit Stauräumen. Dazu erfolgen z. B. im Projekt SEDECO ein Monitoring und eine Modellierung der Entwicklung von in der Natur im Projekt „Thaya2020“ umgesetzten Mäanderanbindungen, die Untersuchung der Möglichkeiten der Relokation von Sedimenten in hochwassergefährdeten Gebieten in Teilbereiche des Stauraums Nové Mlýny als Inselschüttungen oder Maßnahmen zur Förderung der eigendynamischen Seitenerosion zur Verbesserungen der
Ökosystemleistungen. SEDDON II behandelt Teststrecken östlich Wien und im Oberen Abschnitt der ungarischen Donau und DREAM-SK-AT arbeitet in der gemeinsamen Grenzstrecke der Donau.

\subsection{Sedimentaktivitäten im Rhein- und Elbeeinzugsgebiet}

\subsubsection{Rhein}

Österreich hat neben dem absolut größten Anteil am Donauraum ein Teileinzugsgebiet des Rhein. 2018 hat die Internationale Kommission für die Hydrologie des Rheingebietes (https://chrkhr.org/de) erstmals eine Sedimentbilanz publiziert (KHR 2017 (Hillebrand und Frings): Von der Quelle zur Mündung: Die Sedimentbilanz des Rheins im Zeitraum 1991-2010). Darin wird eine detaillierte Sedimentbilanz des Rheins von der Quelle bis zur Mündung präsentiert. Es konnten vier Abschnitte mit divergierendem Sedimenttransportverhalten unterschieden werden, wobei die maximalen Sedimentfrachten im Alpenrhein, flussauf des Bodensees, erreicht werden. Gemäß der Untersuchungen (KHR 2017) verlassen kaum Sedimente den Bodensee und generell weist die Tonfraktion die größten Frachten auf. Kies und Steine werden großteils anthropogen eingetragen. Se- dimentsenken stellen Baggerungen und Ablagerungen in Überflutungsflächen sowie Häfen dar. Während flussauf die Ablagerung dominiert, kommt es flussab verstärkt zur Erosion des Flussbetts. Es wurde festgestellt, dass Sand eine unterschätzte Rolle im Sedimenttransport und der Flussmorphologie des Rheins spielt.

Frings et al. (2014) zeigten, dass der Rhein in verschiedenen Abschnitten signifikante Erosion- und Anlandungstendenzen aufweist, wobei das Rhein-Flussbett z.B. zwischen Iffezheim (Rhein-km 334, $107 \mathrm{~m}$ ü. M.) und flussauf von Königswinter (Rhein-km 646, $44 \mathrm{~m}$ ü. M.) trotz der Geschiebezugabe am oberen Ende ein Disäquilibrium aufweist, mit einer NettoSohleintiefung von $3 \mathrm{~mm} / \mathrm{Jahr}$.

Nur 0,19 Mio. t/Jahr des gesamten Sedimenttransports kommen von flussauf. Der überwiegende Teil stammt von der künstlichen Sedimentzugabe (0,46 Mio. $t / J a h r)$. Ungefähr die gleiche Menge (0,38 Mio. t/Jahr) stammt aus der Sohle infolge der Sohleintiefung (Frings et al. 2014).

Blom et al. (2017) diskutieren die Sohleintiefung in der deutsch-niederländischen Grenzstrecke und starteten grundlegende Untersuchungen zu diesem Thema. Gemäß dieser Untersuchungen hat sich das Flussbett flussauf des niederländischen Rheindeltas über 
das letzte Jahrhundert um 1 bis $2 \mathrm{~m}$ eingetieft.

Als Präsident der KHR hat der Erstautor das Thema Sedimenttransport für das Arbeitsprogramm der kommenden Jahre neben anderen wichtigen Fragestellungen in Hinblick auf die Schaffung einer wissenschaftlichen Grundlage für das verbesserte Management des Rhein vorgeschlagen. Die KHR-Ergebnisse bilden dann die wissenschaftliche Basis für die Arbeit der Internationalen Kommission zum Schutz des Rheins (https://www.iksr.org/de/).

\subsubsection{Elbe}

Die Internationale Kommission zum Schutz der Elbe (IKSE) hat als erstes Flussgebiet ein Sedimentmanagementkonzept für die internationale Flussgebietseinheit Elbe ausgearbeitet (2014, https://www.ikse-mkol.org/).

Von der Arbeitsgruppe der IKSE wurden Vorschläge für eine gute Sedimentmanagementpraxis im Elbegebiet zur Erreichung überregionaler Handlungsziele gemacht. Der Sedimentstatus wurde unter quantitativem, qualitativem und hydromorphologischem Aspekt anhand spezifischer Indikatoren analysiert und bewertet. Als quantitative Indikatoren dienten Schwebstoffkonzentration und Schwebstofffracht. Qualitative Indikatoren waren die im Kontext des Sedimentmanagements relevanten Schadstoffe. Partikelgebundene Schadstoffe stellen ein potenzielles Umweltrisiko dar, wobei 29 Schadstoffe bzw. Schadstoffgruppen als relevant festgestellt wurden und ein unterer und oberer Schwellenwert definiert wurde (IKSE 2014). Schließlich kamen hydromorphologische Indikatoren zur Anwendung (Beeinflussung des Abflussregimes, mittlere Sohlhöhenänderung/Sedimentbilanz, Sedimentdurchgängigkeit, Breitenvarianz/ Tiefenvarianz, Korngrößenverteilung des Sohlsubstrats, Uferstabilität, Uferstruktur, Verhältnis rezente zu morphologischer Aue). Es zeigte sich, dass z. B. an der Binnenelbe ab dem Ende des 19. Jahrhunderts die mittlere Sohlhöhe um bis zu $2 \mathrm{~m}$ eingetieft wurde (IKSE 2014). Das Sedimentdefizit für die gesamte deutsche Binnenelbe beträgt lt. IKSE ca. 0,45 Mio. t/Jahr. Die Risikoanalyse Schifffahrt ergab z.B. für die Binnenelbe, dass die Stabilität wasserwirtschaftlicher Anlagen und die Funktion von Regelungsbauwerken durch hochwasserbedingte Feststoffverfrach- tungen beeinflusst werden. Dies bedingt eine Verschlechterung der Schifffahrtsparameter durch Absinken des Wasserspiegels infolge unzureichender Baggeraktivitäten, eine generell defizitäre Unterhaltung der Regelungsbauwerke und Auswirkungen der Sohleintiefung auf die Fahrrinnenverhältnisse und die Bauwerkssicherheit (IKSE 2014). Betreffend die Sedimentqualität zeigt die Untersuchung der IKSE, dass die oberen Schwellenwerte für viele relevante Schadstoffe an Referenzstellen der Elbe und auch für die Moldau, Mulde und Saale überschritten werden. Im tschechischen Teileinzugsgebiet werden die oberen Schwellenwerte bei mehr als $50 \%$ der relevanten Stoffe bereits in der tschechischen mittleren Elbe überschritten. Es werden von den Autoren auch die Quellentypen dargestellt (Auen und weitere Senken, Punktquellen, Sedimente und Altsedimente u.a. in Buhnenfeldern, Altlasten am Gewässer, sonstige Quellen). Abschließend folgen Vorschläge für einen nachhaltigen Umgang mit Sedimenten und zur Baggergutunterbringung und es wurden Eckpunkte für ein Monitoring zur Gewässerüberwachung und Erfolgskontrolle definiert (IKSE 2014).

\section{Zusammenfassung und Schlussfolgerungen}

International sind umfangreiche Aktivitäten in Zusammenhang mit dem Sedimenttransport festzustellen. Auf der globalen Ebene sind dabei das UNESCO International Hydrological Programme mit der World's Large Rivers Initiative, koordiniert durch den BOKU UNESCO Chair „Integrated River Research and Management“, die International Sediment Initiative, die World Association of Sedimentation and Erosion Resarch, aber auch die wissenschaftlichen Organisationen IAHR, IAHS, IAG oder etwa die ICOLD zu nennen. Dazu gibt es Spezialkonferenzen oder zumindest Sediment Sessions, SCI-Zeitschriften, aber auch Guidelines für die Praxis. Auf europäischer Ebene ist SedNet aktiv und versucht, eine Verbindung zwischen Forschung und Praxis sowie europäischen Richtlinien, Guidelines etc. herzustellen. Auf regionaler Ebene werden im Artikel die Projekte SedAlp und HyMoCARES für den Alpenraum kurz präsentiert. Schließlich erfolgt die Beschreibung einzugsgebietsorientierter Projekte für den Donauraum (DREAM, DanubeSediment, bilaterale Projek- te zwischen Österreich und Ungarn, Slowakei und der Tschechischen Republik) und Aktivitäten im Rhein- und Elbeeinzugsgebiet. Diese Aktivitäten weisen großteils eine Mitwirkung der AutorInnen auf. Es gibt darüber hinaus sicherlich noch weitere sedimentrelevante Aktivitäten, die aber den Rahmen dieses Artikels sprengen würden.

All diesen Gruppierungen und Einrichtungen ist gemein, dass eine $\mathrm{Zu}$ nahme der Aktivitäten im Bereich Sedimenttransport festzustellen ist, was die Bedeutung dieses Themas für ein nachhaltiges Fließgewässermanagement unterstreicht. Gleichzeitig kann festgehalten werden, dass im Bereich der Sedimenttransportprozesse eine Verbesserung des Grundlagenwissens, aber auch der anwendungsorientierten Erkenntnisse notwendig ist. Betreffend das Management entwickelt sich gerade das Bewusstsein für die grundlegende Bedeutung des Sedimenttransports, wobei in Österreich im 2. Nationalen Gewässerbewirtschaftungsplan (NGP) bereits Aussagen zu diesem Thema inkludiert sind und beim 3. NGP eine Weiterführung und Konkretisierung angestrebt werden sollte. Im Donauraum zeichnet sich eine stärkere Beachtung des Themas Sedimente beim nächsten Donau-Flussgebietsbewirtschaftungsplan und Hochwasserrisikomanagementplan, koordiniert durch die ICPDR ab, und eine explizite Aufnahme in die „Significant Water Management Issues“ (eigenständig oder als Teil der „Hydromorphology“) wird vom Projekt DanubeSediment vorgeschlagen. Auf europäischer Ebene wird das Sedimentthema sukzessive Eingang in die Umsetzung der Wasserrahmenrichtlinie und Hochwasserrichtlinie finden. Global wäre es wichtig, einen Überblick über den Status der Flüsse und insbesondere auch den Sedimenthaushalt und -transport zu gewinnen, zumal es sich mehr und mehr zeigt, dass die Sedimente das Rückgrat der Fließgewässer bilden. Die zunehmende Schere zwischen Überschuss und Defizit, verstärkt durch den Klimawandel und die Landnutzungsänderung, beeinträchtigt die Nutzung der Flüsse durch die Wasserkraft oder Schifffahrt, den Schutz vor Hochwässern oder auch den Schutz der Gewässer und der damit verbundenen Ökosysteme. Es zeigt sich sehr deutlich, dass nur eine integrative, nutzungsübergreifende Betrachtung des Sedimenttransports $\mathrm{zu}$ nachhaltigen, technisch umsetzbaren, wirtschaftlich 
und sozial verträglichen und ökologisch positiv wirkenden Verbesserungsmaßnahmen führt.

Danksagung Die Projekte werden durch die Europäische Union im Rahmen des INTERREG-Donauprogramms, der bilateralen EU-Projekte INTERREG AT-HU, INTERREG SK-AT, INTERREG CZ-AT sowie INTERREG IWB und das INTERREG-Alpenraumprogramm kofinanziert. Wir danken unseren Projektpartnern im Ausland sowie in Österreich dem BMNT, dem BMBWF, dem BMVIT, dem BMDW, der Stadt Wien, dem Land NÖ und dem Land OÖ für die finanzielle Unterstützung. Weiters bedanken wir uns bei viadonau, dem Verbund und dem HD Oberösterreich für die Bereitstellung der notwendigen Daten. Wir bedanken uns für die finanzielle Unterstützung durch die Christian Doppler Forschungsgesellschaft, das Bundesministerium für Digitalisierung und Wirtschaftsstandort und die Nationalstiftung für Forschung, Technologie und Entwicklung.

Funding Open access funding provided by University of Natural Resources and Life Sciences Vienna (BOKU).

Open Access Dieser Artikel wird unter der Creative Commons Namensnen- nung 4.0 International Lizenz (http:// creativecommons.org/licenses/by/4. $0 /$ deed.de) veröffentlicht, welche die Nutzung, Vervielfältigung, Bearbeitung, Verbreitung und Wiedergabe in jeglichem Medium und Format erlaubt, sofern Sie den/die ursprünglichen $\mathrm{Au}$ tor(en) und die Quelle ordnungsgemäß nennen, einen Link zur Creative Commons Lizenz beifügen und angeben, ob Änderungen vorgenommen wurden.

Hinweis des Verlags Der Verlag bleibt in Hinblick auf geografische Zuordnungen und Gebietsbezeichnungen in veröffentlichten Karten und Institutsadressen neutral.
Aigner, J., Kreisler, A., Haimann, M., Rindler, R., Sindelar, C., Habersack, H., Pichler, A (2014): SedAlp - Sedimentmanagement in alpinen Einzugsgebieten Österreichische Wasserund Abfallwirtschaft, 9-10/14, 348-356; ISSN 0945-358X

Annandale, G. W., Randle, T. J., Langendoen, E. J. Hotchkiss, R. H and The United States National Reservoir Sedimentation and Sustainability Team (NRSST) (2018): Hydrolink 3, 72-75

Best, J. (2019): Anthropogenic stresses on the world's big rivers Nature Geoscience, VOL 12 , $7-21$

Blom, A., L. Arkesteijn, V. Chavarrías, and E. Viparelli (2017): The equilibrium alluvial river under variable flow and its channel-forming discharge, J. Geophys. Res. Earth Surf., 122, 1924-1948, https://doi.org/10.1002/2017JF00 4213

Bock, N., Gökler, G., Reindl, R., Reingruber, J., Schmalfuß, R., Badura, H., Frik, G., Leobner, I., Lettner, L., Scharsching, M., Spreitzer, R., Thöni, M. (2019): Feststoffmanagement be Wasserkraftanlagen in Österreich, Kanal. Österreichische Wasser- und Abfallwirtschaft, 71. DanubeSediment 2019: Report of WP3, geleitet von BME/HU

DanubeSediment 2019: WP4, geleitet von VUVH SK.

Dogterom, J. (2001): Assessment practices and environmental status of 10 transboundary river in Europe, International Water Assessment Centre under the UNECE Convention on Protection and Use of Transboundary Watercourses and International Lakes. Helsinki, 1992 International water assessment Centre/Riza (2001), $141 \mathrm{~S}$.

Frings, R. M., Gehres, N., Promny, M., Middelkoop, H., Schüttrumpf, H., Vollmer, S. (2014) Today's sediment budget of the Rhine River channel, focusing on the Upper Rhine Graben and Rhenish Massif, Geomorphology 204 573-587.

Gupta, A. (2008): Large Rivers: Geomorphology and Management (Wiley and Sons, Chichester). Habersack, H., BMLFUW, Pucher, K., Schober, B, Samek, R., Hackel, C. (2015): Hochwasserdokumentation Donau 2013. Ereignisdokumentation, Universität für Bodenkultur Wien.

Habersack, H., Hein, T., Stanica, A., Liska, I. Mair, R., Jager, E., Hauer, C., Bradley, C. (2016) Challenges of river basin management: Current status of, and prospects for, the River Danube from a river engineering perspective. SCI TOTAL ENVIRON. 2016; 543: 828-845.
Habersack, H., Huber-Humer, M., Wagner, B., Mostbauer, P., Hrad, M., Fuhrmann, M., Aigner, J., Klösch, M. (2018): Qualität von Räummaterial aus Wildbächen: Entwicklung einer Methodik für ein vereinfachtes Prüfverfahren. Endbericht. Studie im Auftrag des Bundesministeriums für Nachhaltigkeit und Tourismus, Abteilung III/5, Wildbach- und Lawinenverbauung, $180 \mathrm{~S}$.

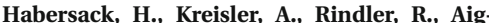
ner, J., Seitz, H., Liedermann, M., Laronne, J. B. (2017): Integrated automatic and continuous bedload monitoring in gravel bed rivers, GEOMORPHOLOGY. 2017; 291: 80-93.

Hauer, C., Wagner, B., Aigner, J., Holzapfel, P. Flödl, P., Liedermann, M., Tritthart, M., Sindelar, C., Klösch, M., Haimann, M., Habersack, H. (2019): Das „Christian Doppler Labor fü Sedimentforschung und -management": Anwendungsorientierte Grundlagenforschung und Herausforderungen für eine nachhaltige Wasserkraft und Schifffahrt, Österreichische Wasserund Abfallwirtschaft, 71

http://isrs2019.info/cms/index.php/home-235. html (Zugriff 15.01.2019)

http://worldslargerivers.boku.ac.at/wlr/ (Zugriff 15.01.2019)

http://www.geomorph.org/ (Zugriff 15.01.2019) http://www.isrs2019.cn/ (Zugriff 15.01.2019) http://www.waser.cn/waser/index.htm (Zugriff 15.01.2019)

https://chr-khr.org/de (Zugriff 15.01.2019)

https://en.unesco.org/themes/water-security/

hydrology (Zugriff 15.01.2019)

https://iahs.info (Zugriff 15.01.2019)

https://limnology.org/ (Zugriff 15.01.2019)

https://sednet.org/ (Zugriff 15.01.2019)

https://sites.agu.org/ (Zugriff 15.01.2019)

https://www.alpine-space.eu/projects/hymocares/ en/home (Zugriff 15.01.2019)

https://www.egu.eu/ (Zugriff 15.01.2019)

https:/ / www.hydropower.org/ (Zugriff 15.01.2019)

https://www.iahr.org/ (Zugriff 15.01.2019)

https://www.icold-cigb.org/ (Zugriff 15.01.2019)

https://www.ikse-mkol.org/ (Zugriff 15.01.2019)

https://www.iksr.org/de/ (Zugriff 15.01.2019)

https://www.journals.elsevier.com/international-

journal-of-sediment-research (Zugriff 15.01.2019)

HyMoCARES 2019: Project report

Internationale Kommission zum Schutz der El-

be (IKSE) 2014: Sedimentmanagementkonzep

der IKSE Vorschläge für eine gute Sedimentma-

nagementpraxis im Elbegebiet zur Erreichung

überregionaler Handlungsziele.
KHR (2017): Bericht Nr. II-22 der KHR (ed. Hillebrand \& Frings), KHR/CHR ISBN: 978-90-7098039-9 https://doi.org/10.5675/KHR_22.2017.

Kloesch, M., Habersack, H. (2017): The Hydromorphological Evaluation Tool (HYMET). GEOMORPHOLOGY; 291: 143-158.

Kondolf, G. M., Schmitt, R. J. P., Carling, P., Darby, S., Arias, M., Bizzi, S., Castelletti, A., Cochrane, T. A., Gibson, S., Kummu, M., Oeurng, C. Rubin, Z., Wild, T. (2018): Changing sedimen budget of the Mekong: Cumulative threats and management strategies for a large river basin. Science of The Total Environment.

Liedermann, M., Aigner, J., Kreisler, A., Klösch, M., Rindler, R., Gmeiner, P., Pessenlehner, S. Tritthart, M., Hauer, C., Habersack, H. (2019): Forschung zu alpinen Sedimentprozessen als Basis für ein verbessertes Feststoffmanagement unter Berücksichtigung extremer Ereignisse. Österreichische Wasser- und Abfallwirtschaft, 71.

Nocquet, J.-M. C. Sue, A. Walpersdorf, T. Tran, N. Lenôtre, P. Vernant, M. Cushing, E Jouanne, F. Masson, S. Baize, J. Chéry \& P. A. van der Beek (2016): Present-day uplift of the western Alps. Sci. Rep. 6, 28404; https://doi.org/10.1038/ srep28404.

Schobesberger, J., Lehmann, T., Kimmersdor fer, A., Lichtneger, P., Sindelar, C., Habersack, H. (2019): Urbanes Sedimentmanagement vom Einzugsgebiet bis zum Kanal. Österreichische Wasser- und Abfallwirtschaft, 71 https:// doi.org/10.1007/s00506-018-0551-z

Schwarz, U., Babic-Mladenovic, M., Bondar, C. Gergov, G., Holubova, K., Modev, S., Rákóczi, L., Rast, G., Steindl, J., Sorin, T., Anna, T.E. (2008): Assessment of the balance and management of sediments of the Danube waterway, curren status, problems and recommendations for actions. World wide Fund for Nature, Vienna (Pub. $59 \mathrm{pp}$.).

SedNet (2017): Effective river basin management needs to include sediment, policy brief.

Skolaut, C., Liébault, F., Habersack, H., Lenzi, M., Rusjan, S., Sodnik, J., Pichler, A. (2015): Synthesis report of the project SedAlp - Sediment management in Alpine basins: integrating sediment continuum, risk mitigation and hydropower, $36 \mathrm{~S}$.

Stanica, A., Panin, N. (2009): Present evolution and future predictions for the deltaic coastal zone between the Sulina and Sf. Gheorghe Danube river mouths (Romania). Geomorphology 107, 41-46. 


\section{Originalarbeit}

\begin{abstract}
Tritthart, M., Glock, K., Glas, M., Yücesan, S. Liedermann, M., Gmeiner, P., Hauer, C., Habersack, H. (2019): Erfahrungen in der numerischen Sedimenttransportmodellierung auf unterschiedlichen Skalen - von RANS bis LES.

Österreichische Wasser- und Abfallwirtschaft,

71. https://doi.org/10.1007/s00506-018-0550-0
\end{abstract}

Walling, D. E. (2008): The changing sediment loads of the world's rivers. Proceedings of the International Symposium on Sediment Dynamics in Changing Environments, Christchurch, New Zealand. Wallingford UK, IAHS Press IAHS Publication 325, pp. 325-38.
Walling, D. E. (2015): The changing sediment load of the world's rivers and implications for land-ocean sediment fluxes. In Proc. Int. Hydraulic Engineering Symposium Aachen (IWA-

SA) (Aachen, Denmark)

www.sedalp.eu/ (Zugriff 15.01.2019) 